\title{
Spacetime from Zitterbewegung
}

\author{
Mohamed S. El Naschie \\ Dept. of Physics, Faculty of Science, University of Alexandria, Alexandria, Egypt \\ Email: Chaossf@aol.com
}

How to cite this paper: El Naschie, M.S. (2017) Spacetime from Zitterbewegung. Open Journal of Modelling and Simulation, 5, 169-173.

https://doi.org/10.4236/ojmsi.2017.53012

Received: June 13, 2017

Accepted: June 20, 2017

Published: June 23, 2017

Copyright $\odot 2017$ by author and Scientific Research Publishing Inc. This work is licensed under the Creative Commons Attribution International License (CC BY 4.0).

http://creativecommons.org/licenses/by/4.0/ (c) (i) Open Access

\begin{abstract}
Quantum particles are assumed to have a path constituting a random fluctuation super imposed on a classical one resulting in a golden mean spiral propagating in spacetime. Consequently, the dimension of the path of the quantum particle is given by one plus the random Cantor set Zitterbewegung, i.e. $1+\phi$ where $\phi$ is the golden mean Hausdorff dimension of a random Cantor set. Proceeding in this way, we can derive the basic topological invariants of the corresponding spacetime which turned out to be that of E-infinity spacetime $4+\phi^{3}$ as well as a fractal Witten's M-theory $11+\phi^{5}$. Setting $\phi^{3}$ and $\phi^{5}$ equal zero, we retrieve Einstein's spacetime and Witten's M-theory spacetime respectively where $\phi^{3}$ is the latent Casimir topological pressure of spacetime and $\phi^{5}$ is Hardy's quantum entanglement of the same.
\end{abstract}

\section{Keywords}

Zitterbewegung, E-Infinity Theory, Quantum Physics, Einstein Spacetime, Fractal Spacetime, Witten Spacetime, 'tHooft Cellular Automaton

\section{Introduction}

There is an excellent model for Zitterbewegung due to Arend Niehaus, University of Utrecht Physics Professor [1]. This model interpreted in the right way leads to a general theory which goes a long way towards a quantum physics without quantum mechanics or at least without the orthodox form of quantum mechanics [2] [3] [4]. The present short note builds upon this work and shows that by means of minimal number theoretical adjustment, Niehaus's model leads to the basic conclusions of E-infinity Cantorian-fractal spacetime theory [5] and related models [5]-[20]. In particular, we can derive the exact Hausdorff dimension of spacetime and show how it arises naturally from the basic Zitterbewegung model mentioned above [1]. Even more importantly, the present note restores the meaning of a path to quantum physics once we extend the meaning 
beyond that of a one dimensional line of a propagating point particle [5] [6] as will be shown in what follows.

\section{Analysis}

As mentioned in the present Introduction earlier on, the basic idea of the Niehaus Zitterbewegung model [1] is to replace the ordinary path of a classical particle $(d=1)$ by a spiralling line representing a path and a spin simultaneously. Therefore, we could say with reasonable justification that the model is a second generation development of the area-like quantum particle path introduced probably for the first time by Abbot and Wise [5] [6] and subsequently integrated into the fractal spacetime theory of G. Ord and sometime later, L. Nottale [5] and later still by the present author in his E-infinity Cantorian spacetime theory [5]. However there is a crucial difference between the Cantorian area-like path proposal [5] and all other proposals, namely that in E-infinity theory, the "quantum" path of the "quantum" particle is described not by a single Hausdorff or topological dimension, but rather with two inter-dependent dimensions [5]. More precisely in E-infinity theory we use both dimensions simultaneously, namely the topological dimension $(d=2)$ as well as the corresponding Hausdorff dimension exactly as in the continuous geometry model of von Neumann-Connes as applied to Penrose fractal tiling universe [5]-[12] which represents more over a generic case of a specific noncommutative geometry [5]. It is easily shown using the corresponding dimensional function of von NeumannConnes [5] or the equivalent bijection formula of E-infinity that for $(d=2)$ topological dimension of a "quantum" particle, the Hausdorff dimension is $d=1+\phi$ where $\phi=(\sqrt{5}-1) / 2$ is the golden mean, also used extensively in [7]. In the notation of E-infinity this means [5] [8]

$$
d_{c}^{(n=2)}=(1 / \phi)^{n-1}=1 / \phi=1+\phi
$$

Now starting from Newtonian three dimensional classical space, we see that the corresponding dimension must be the triadic intersection given by

$$
D=(1 / \phi)(1 / \phi)(1 / \phi)=(1 / \phi)^{3}=4+\phi^{3}
$$

This is clearly the Hausdorff dimension of a $d=4$ Einstein spacetime as given by the bijection formula [5]

$$
d_{c}^{(n)}=(1 / \phi)^{n-1}
$$

so that $n=4$ leads to [5] [8]

$$
d_{c}^{(4)}=(1 / \phi)^{3}=4+\phi^{3}=4+\frac{1}{4+\frac{1}{4+\cdots}}=4.23606799
$$

The connection to Zitterbewegung of the Niehaus model and the associated theory [1] is as follows.

It is a well known mathematical-geometrical fact that except for the straight line and the perfect circle, only the logarithmic spiral is infinitely self similar homogeneous. In addition the spiral in two dimensions arises from the con- 
struction of a random one dimensional Cantor dust (set) with uniform distribution [12]. The Hausdorff dimension of such a set, as shown long ago in a work by D. Mauldin in a paper dedicated to S. Ulam, is given by the golden mean $\phi$ [5]. Consequently our Hausdorff dimension $1+\phi$ which corresponds to topological dimensions equal two, captures the number theoretical essence of E-infinity theory as well as the noncommutative quantum nature of Connes geometry plus Niehaus Zitterbewegung model all in one maybe lucky stroke although we think it is very unlikely that all that has much to do with luck and is essentially the unreasonable effectiveness of pure mathematics [12].

Before concluding this section let us show using the above result a remarkable derivation connecting superstrings $(d=10)$ with Kaluza-Klein spacetime $(d=5)$ and Witten's M-theory $(d=11)$ [8]. To do that let us send Niehaus Zitterbewegung to Kaluza-Klein spacetime $(d=5)$. That way we find [8]

$$
D=(1 / \phi)^{5}=11+\phi^{5}
$$

where $\phi^{5}$ is the exact Hardy quantum entanglement probability of two quantum particles [8]. Setting $\phi^{5} \simeq$ zero compared with 11 , one finds the original non-fractal M-theory of Witten [8]. In fact $11+\phi^{5}$ is the Hausdorff dimension corresponding to the Calabi-Yau compactified section of $d=10$ superstrings because $d=10-4=6$ [5] [8] and we have therefore

$$
d_{c}^{(6)}=(1 / \phi)^{6-1}=(1 / \phi)^{5}=11+\phi^{5}
$$

with which we conclude this compressed, very short analysis. For in depth study of the ideas and theories discussed here, the reader is directed to Refs. [9]-[11] and as far as E-infinity theory is concerned, Refs. [12]-[20] are quite valuable.

\section{Conclusions}

The effort of what might be called the Utrecht Dutch School of G. 'tHooft in inventing or discovering a quantum physics without the unintuitive and/or at least classically paradoxical orthodox quantum mechanics seems to have some considerable success by the non-mainstream efforts of people like Gerard 'tHooft himself [9] and recently Niehaus Zitterbewegung theory [1]. These efforts are by no means confined to the work carried out in Holland [9] and there are various schools all over the world seriously engaged in the dream of restoring at least partially, some of our Newtonian classical intuition and common sense to modern quantum physics and cosmology, for instance by S. Weinberg [10] [11]. We hasten to say that it would be philosophically misguided to think that matrix quantum mechanics, Schrödinger and Dirac's equations, let alone the marvellous quantum field theory did not bring to us a considerable understanding of nature or that it was all a historical accident, not more. It is just the contrary because it was these methods and theories [11] which made an almost exact prediction but failed to satisfy our deep seated human need or urge for understanding which compelled us to travel all possible roads and to climb all possible mathematical and theoretical physics mountains [11] [12] to see the horizon at infinity [11]. It is may be as expressed by Niels Bohr, thegreat Dane in astoni- 
shingly eloquent German [12].

"Nur in der fuller Liegt die Klarkeit", i.e. only in the abundance lies clarity.

This does not only apply to the physical phenomena but also to the mathematical models and theories which we apply [12].

\section{References}

[1] Niehaus, A. (2017) Zitterbewegung and the Electron. Journal of Modern Physics, 8, 511-521. https://doi.org/10.4236/jmp.2017.84033

[2] El Naschie, M.S. (2007) On Gauge Invariance, Dissipative Quantum Mechanics and Self Adjoint Sets. Chaos, Solitons \& Fractals, 32, 271-273.

[3] El Naschie, M.S. (1995) A Note on Quantum Mechanics, Diffusional Interference and Information. Chaos, Solitons \& Fractals, 5, 881-884.

[4] El Naschie, M.S. (2007) Deterministic Quantum Mechanics versus Classical Mechanical Indeterminism and Nonlinear Dynamics. American Institute of Physics, AIP Conference Proceedings No. 905, May 2007, New York, 56-66.

[5] El Naschie, M.S. (2004) A Review of E-Infinity and the Mass Spectrum of High Energy Particle Physics. Chaos, Solitons \& Fractals, 19, 209-236.

[6] Abbot, L.F. and Wise, M.B. (1981) Dimensions of a Quantum Path. American Journal of Physics, 49, 37-39. https://doi.org/10.1119/1.12657

[7] Jan, C.A. (2013) Boeyens: The Chemistry of Matter Waves. Springer, Berlin, Germany.

[8] El Naschie, M.S. (2016) On a Fractal Version of Witten's M-theory. Journal of Astronomy \& Astrophysics, 6, 135-144.

[9] 'tHooft, G. (2016) The Cellular Automaton Interpretation of Quantum Mechanics. Springer Open (Springer International Publishing, Germany), Berlin.

https://doi.org/10.1007/978-3-319-41285-6

[10] Weinberg, S. (2014) Quantum Mechanics without State Vectors. http://arxiv.org/pdf/1405.3483v1.pdf

[11] Weinberg, S. (2015) To Explain The World: The Discovery of Modern Science. Harper, New York, USA.

[12] El Naschie, M.S. (2016) Einstein's Dark Energy via Similarity Equivalence, 'tHooft Dimensional Regularization and Lie Symmetry Groups. International Journal of Astronomy \& Astrophysics, 6, 56-81. https://doi.org/10.4236/ijaa.2016.61005

[13] El Naschie, M.S. (2017) The Looped Light of the Triple-Slit Real Experiment as a Confirmation for the Extra Dimensions of Quantum Spacetime and the Reality of Dark Energy. Optical and Photonic Journal, 7, 19-26. https://doi.org/10.4236/opj.2017.72003

[14] El Naschie, M.S. (2017) Looped Light on Dark Energy. Journal of Quantum Information Science, 7, 43-47. https://doi.org/10.4236/jqis.2017.72004

[15] El Naschie, M.S. (2017) The Quantum Triple-Slit Experiment and Dark Energy. Open Journal of Microphysics, 7, 31-35. https://doi.org/10.4236/ojm.2017.72002

[16] El Naschie, M.S. (2017) Quantum Disentanglement as the Physics Behind Dark Energy. Open Journal of Microphysics, 7, 1-27.

https://doi.org/10.4236/ojm.2017.71001

[17] El Naschie, M.S. (2017) Kähler Dark Matter, Dark Energy, Cosmic Density and Their Coupling. Journal of Modern Physics, 7, 1953-1962.

[18] El Naschie, M.S. (2016) Completing Einstein's Spacetime. Journal of Modern Phys- 
ics, 7, 1972-1994. https://doi.org/10.4236/jmp.2016.715175

[19] El Naschie, M.S. (2016) High Energy Physics and Cosmology as Computation. American Journal of Computational Mathematics, 6, 185-199. https://doi.org/10.4236/ajcm.2016.63020

[20] El Naschie, M.S. (2005) On a Fuzzy Kähler-Like Manifold Which Is Consistent With The Two Slit Experiment. International Journal of Nonlinear Sciences and Numerical Simulation, 6, 95-98. https://doi.org/10.1515/IJNSNS.2005.6.2.95

Submit or recommend next manuscript to SCIRP and we will provide best service for you:

Accepting pre-submission inquiries through Email, Facebook, LinkedIn, Twitter, etc. A wide selection of journals (inclusive of 9 subjects, more than 200 journals)

Providing 24-hour high-quality service

User-friendly online submission system

Fair and swift peer-review system

Efficient typesetting and proofreading procedure

Display of the result of downloads and visits, as well as the number of cited articles Maximum dissemination of your research work

Submit your manuscript at: http://papersubmission.scirp.org/

Or contact ojmsi@scirp.org 